\title{
Special issue on "drones as enablers of novel services: operational and technology challenges"
}

\author{
Ana M. Bernardos ${ }^{1} \cdot$ Juan A. Besada ${ }^{1}$. Jesús García Herrero ${ }^{2} \cdot$ Hideo Saito $^{3} \cdot$ Patrizia Marti $^{4}$ \\ Published online: 14 March 2021 \\ (C) The Author(s), under exclusive licence to Springer-Verlag London Ltd. part of Springer Nature 2021
}

\section{Introduction}

Unmanned Aerial Vehicles (UAVs) - based applications and services are no more a remote possibility but an economic and social opportunity. Emergency-response coordination, infrastructure monitoring, urban planning, security, logistics or support for intelligent transportation systems are only some of the domains in which UAVs may become key in the near future. In particular, drones-in-the-city will become aerial sensing platforms that will be integrated into urban sensing networks, and the data they generate will be merged with the data flow coming from existing sensors through IoT platforms, to deliver new services and support innovative business models. Apart from the legislative changes needed to make urban drone-based applications feasible, it is essential to ensure the efficient and safe operation of UAV fleets within a restrictive and complex operating area. It is also important to enable the accurate acquisition, secure transmission and correct interpretation of drone-gathered information to allow new applications and business models to develop.

This Special Issue aims to bring together contributions that examine the operational, business and technology challenges that the use of drones poses. Eight articles cover different

Ana M. Bernardos

abernardos@grpss.ssr.upm.es

1 Information Processing and Telecommunications Center, Universidad Politécnica de Madrid, ETSI Telecomunicación, Av. Complutense 30, 28040 Madrid, Spain

2 Universidad Carlos III de Madrid, Campus de Colmenarejo, Avd. de Gregorio Peces-Barba Martínez, 22, 28270 Colmenarejo, Madrid, Spain

3 Keio University, Faculty of Science and Technology, Yagami Campus, 3-14-1 Hiyoshi, Kohoku-ku, Yokohama, Kanagawa 223-8522, Japan

4 University of Siena, Via Roma 56, 53100 Siena, Italy topics related to operations planning, service provision, examples of services over drones (communications and monitoring) and UAV technology specificities (such as navigation improvement and drone image leverage). Following there is a brief summary of the specific contents of each article.

In "Drone Flight Planning for Safe Urban Operations", by Juan A. Besada et al., it is described the requirements of a flight planning tool for safe urban operations, which may be used to design operations considering flight constraints and limitations. Additionally, authors show how the system is designed to work in coordination with an unmanned traffic management system (UTM) in charge of distributing available very low-level airspace resources among different operations and authorizing them, and of monitoring compliance of actual flights with flight authorizations.

In "A control and communications platform for procedural mission planning with multiple aerial drones", by Bruno Areias et al., authors describe a modular solution for multi-drone control that implements the functionality required for planning, execution, and monitoring of procedural, multi-step missions with optional drone cooperation. The performance of drone-to-ground communications, with a particular focus on latency, is evaluated, together with the performance of commercial built-in message brokers, which seem not be suitable for drone swarms.

"Drones-as-a-Service: a Simulation-Based Analysis for OnDrone Decision Making", by Majed Alwateer et al., includes an analysis on how a variety of drone applications (security, logistics and transport, surveillance, etc.) can be delivered as a service, using a common set of underlying service provisioning principals, such as making decisions about who to serve next. Authors also study by simulation how different decision-making strategies on drone flights impact clients and service providers.

In "On-Demand UAV Base Station Deployment for Wireless Service of Crowded Tourism Areas", Lijie Yin et al. propose an on-demand coverage solution based on unmanned aerial vehicle (UAV) base stations to deal with the high demand that specific tourism-related events may require. The methodology includes defining the effective coverage 
radius, based on which the optimal altitude of UAV BS is derived, to then tackle the inherent challenge of irregular tourist flow through an automatic UAV BS deployment algorithm.

In "SwarmCity project: monitoring traffic, pedestrians, climate and pollution with an aerial robotic swarm", Juan Jesús Roldán et al., propose a robotic swarm to collect the data of traffic, pedestrians, climate, and pollution. Data are sent to a base station, where they are treated to generate maps and presented in an immersive interface. To validate these developments, authors use a virtual city called SwarmCity with models of traffic, pedestrians, climate, and pollution based on real data. Results show that the complete solution - i.e., fleet control, data fusion, and operator interface - allows monitoring the relevant variables in the simulated city.

Different technology issues related to UAV performance and capabilities are considered in the next three articles. "Simulation in real conditions of navigation and obstacle avoidance with PX4/Gazebo platform", by Jesús García et al., presents an approach to integrate and evaluate a LIDAR sensor and the capacity for improving navigation and obstacle avoidance functions in simulated situations using a real UAV platform. The proposed solution (a general method that could be used to integrate other kind of sensors) shows physical integration of the main types of sensors in UAV domain both for navigation and collision avoidance, and at the same time the use of powerful simulation models developed with Gazebo.

In "A Superresolution Enhancement of UAV Images based on a Convolutional Neural Network for Mobile Devices", Miguel A. Patricio et al., focus on how to reconstruct highresolution images given low-resolution images (image superresolution, SR), proposing an architecture for the SR of images captured by a UAV. The images are sent from a lowresolution camera in a UAV to a mobile device from which it is possible to obtain the corresponding SR image. A benchmark dataset of images is used for both quantitative and qualitative assessment.

"Aerial Image Super-Resolution based on Deep Recursive Dense Network for Disaster Area Surveillance", by Feiqiang Liu et al., also tackle the production of high-resolution (HR) aerial images, in particular to monitor a disaster area from the air. Authors propose a deep recursive dense network (DRDN) to reconstruct HR aerial images. In the DRDN, the proposed recursive dense block (RDB) can fully extract abundant local features and adaptively fuse different hierarchical features of LR image for HR image reconstruction. In addition, the recursive manner of RDB in DRDN can effectively reduce the parameter of network. The experimental results on aerial images demonstrate the superiority of our proposed method.

All in all, the compiled articles show the evolution of some hot topics in nowadays research related to drones and droneenabled systems. In particular, it is interesting that some of the works are focused on delivering operational tools and services over urban spaces, which are assumed to be key to leverage the potential of UAV in terms of services. A lot of open issues (related to traffic management, safety, privacy, pollution, urban impact, user acceptance, etc.) are yet to be evaluated and explored to make drones-based applications a reality in these scenarios.

As final remark, guest editors want to heartfully thank Prof. Peter Thomas, PUC Editor-in-Chief, for his support; authors, for submitting their contributions; referees, for providing their thoughtful insights and comments to select and improve the manuscripts, and the staff of the journal's Editorial Office and Production Department for all the help.

\section{Guest Editors}

- Ana M. Bernardos, Universidad Politécnica de Madrid, ETSI Telecomunicación, Av. Complutense 30, 28,040 Madrid, Spain.

- Juan A. Besada, Universidad Politécnica de Madrid, ETSI Telecomunicación, Av. Complutense 30, 28,040 Madrid, Spain.

- Jesús García Herrero, Universidad Carlos III de Madrid, Campus de Colmenarejo Avd. de Gregorio Peces-Barba Martínez, 2,228,270 Colmenarejo, Madrid, Spain.

- Hideo Saito, Keio University, Faculty of Science and Technology, Yagami Campus

3-14-1 Hiyoshi, Kohoku-ku, Yokohama, Kanagawa 223-8522, Japan.

- Patrizia Marti, University of Siena, Via Roma 56, 53,100 Siena, Italy.

\section{Paper order}

- Drone Flight Planning for Safe Urban Operations

- A control and communications platform for procedural mission planning with multiple aerial drones

- Drones-as-a-Service: a Simulation-Based Analysis for On-Drone Decision Making

- On-Demand UAV Base Station Deployment for Wireless Service of Crowded Tourism Areas

- SwarmCity project: monitoring traffic, pedestrians, climate and pollution with an aerial robotic swarm

- Simulation in real conditions of navigation and obstacle avoidance with PX4/Gazebo platform

- A Superresolution Enhancement of UAV Images based on a Convolutional Neural Network for Mobile Devices

- Aerial Image Super-Resolution based on Deep Recursive Dense Network for Disaster Area Surveillance

Publisher's note Springer Nature remains neutral with regard to jurisdictional claims in published maps and institutional affiliations. 